\title{
Integrated multiple transcriptomes in oviductal tissue across the porcine oestrous cycle reveal functional roles in oocyte maturation and transport
}

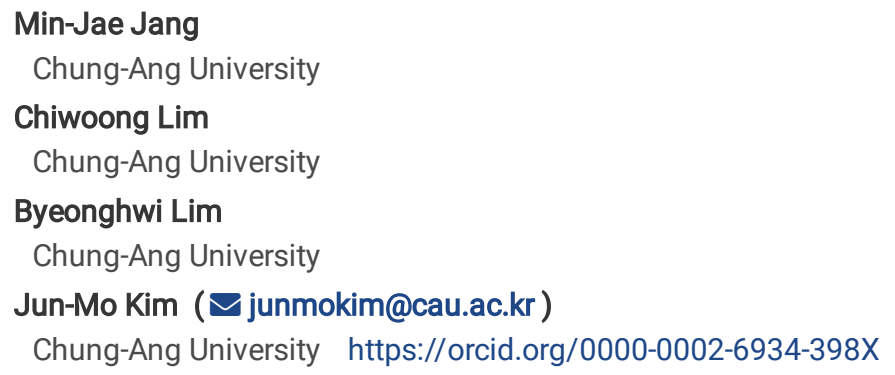

\section{Research}

Keywords: Pig oestrous cycle, RNA-seq, DEG, Reproductive tissue

Posted Date: August 2nd, 2021

DOI: https://doi.org/10.21203/rs.3.rs-763058/v1

License: (1) This work is licensed under a Creative Commons Attribution 4.0 International License. Read Full License

Version of Record: A version of this preprint was published at Journal of Animal Science on December 16th, 2021. See the published version at https://doi.org/10.1093/jas/skab364. 


\section{Abstract \\ Background}

Understanding the complex changes in the swine female reproductive system is important for solving issues related to reproductive failure as well as the litter size. The oviduct is the site of fertilization, from where the fertilized egg moves to the uterus for implantation. Hence, elucidating the regulatory mechanisms of the natural oestrous cycle in the oviduct under non-fertilization conditions can improve our understanding of their roles in the reproductive system.

\section{Results}

In this study, whole transcriptome RNA sequencing of oviduct tissue samples was performed on Days $0,3,6,9,12,15$, and 18 across the entire oestrous cycle to screen for differentially expressed genes (DEGs). We identified 7,623 DEGs across all the time points relative to Day 0 . The DEGs were distinctly classifiable into three major clusters according to their expression patterns. Clusters 1 and 2 (1,222 and 1,146 genes, respectively) included genes involved in overall physiological changes observed through the oestrous cycle. Cluster 1 included genes were mainly involved in PI3K-Akt signalling and steroid hormone biosynthesis pathways, while Cluster 2 genes were involved in extracellular matrixreceptor interaction and protein digestion pathways. The expression levels of Cluster 3 genes were specifically downregulated in the luteal phase. We constructed a network with 1,000 Cluster 3 genes. KEGG pathway enrichment analyses revealed that the DEGs in Cluster 3 were strongly associated with cell cycle, calcium signalling, and oocyte meiosis. According to gene set enrichment analysis, genes associated with calcium signalling pathways and oocyte meiosis were also significantly downregulated in the luteal phase.

\section{Conclusions}

In this study, we identified that the expression of genes in the oviduct during the oestrous cycle affects oocyte transport and fertilization, which are the key functions of the oviduct. Current study provides a basis for successful breeding in the pig industry and uncovers an overall mechanism change for the pig oviduct in the oestrous cycle.

\section{Background}

Reproductive traits of pigs, including litter size, litters per sow per year, and size of the piglets, are important factors in establishing breeding goals [1]. Thus, a comprehensive understanding of the molecular pathways occurring in reproductive tissues during the oestrous cycle is important for solving complications related to the reproductive problems, such as infertility or stillbirths, and consequently controlling the total production per sow. The changing levels of various steroid hormones during an oestrous cycle induce rapid physiological and morphological changes in the female reproductive organs [2].

The swine oestrous cycle lasts about 21 days. As the follicle grows, it produces oestrogen, which reaches its peak level shortly before ovulation Luteinizing hormone ( $\mathrm{LH}$ ) and gonadotropin-releasing hormone levels are also increased as they are involved in a positive feedback loop with oestrogen; however, production of follicle stimulating hormone is inhibited by oestrogen to prevent the maturation of other follicles [3]. Peaking $\mathrm{LH}$ level promotes ovulation and facilitates the release of progesterone from the corpus luteum (CL). Fertilization occurs when an oocyte encounters sperm at the ampullary-isthmic junction. During pregnancy, progesterone continues to be secreted; however, the uterus secretes prostaglandin F2a, which inhibits progesterone secretion from CL [4]. The organs involved in female pig reproduction include the ovary, oviduct, uterus, and endometrium, among which the oviduct, the site of fertilization, is crucial as a hormone-sensitive bridge [5].

To understand the genetic landscape of the oviduct, most studies have used microarray and candidate genes for analysing gene expression patterns and variations [6]. However, a recently developed, high-throughput, data-based technology-next-generation sequencing-enables quantitative analysis of dynamic transcriptomes [7-9]. To date, several studies have conducted whole transcriptome analyses to elucidate the molecular mechanisms in reproductive tissues. Based on correlation, between integrated transcriptomes, Kim et al. (2018) [10] reported that tissue synchronization occurs among three important reproductive organs (the ovary, oviduct, and endometrium). Fischer et al. (2015) [11]also identified nonsynonymous mutations in the genes overexpressed in the testis and oviduct, providing a list of candidate biomarkers of reproductive traits in swine. However, the dynamic changes in oviduct transcriptomes during the oestrous cycle are not yet entirely understood. Therefore, the aim of this study was to determine the time-serial transcriptome profile and the molecular mechanisms occurring in the porcine oviduct during the oestrous cycle using RNA sequencing (RNA-Seq). We explored the differentially expressed genes (DEGs) at each time point and integrated transcriptome profiles to identify gene expression patterns and their modulations.

\section{Materials And Methods}




\section{Animals and experimental design}

Twenty-two crossbred (Landrace $\times$ Yorkshire) gilts aged between 6-8 months, weighing 100-120 kg, and having had at least two oestrous cycles of normal duration (18-22 days) were used in this study. The gilts' oestrous behaviour was observed daily in the presence of a boar according to a previously described method [12]. The first day of oestrous behaviour was designated as Day 0. Oviduct tissues were collected from the ampulla region on Days $0(n=3), 3(n=3), 6(n=2), 9(n=3), 12(n=4), 15(n=4)$, and $18(n=3)$ (Fig. 1A). The collected oviducts were snap-frozen in liquid nitrogen and stored at $-80^{\circ} \mathrm{C}$ for RNA extraction.

All animal-related experimental procedures were performed in accordance with the Guide for Care and Use of Animals in Research and approved by the Institutional Animal Care and Use Committee of the National Institute of Animal Science (No. 2015 - 137).

\section{RNA isolation, library construction, and sequencing}

Total RNA was isolated from oviduct tissue using TRIzol reagent (Invitrogen, Carlsbad, CA, USA) following the manufacturer's protocols [13]. The RNA was quantified using a NanoDrop ND-1000 spectrophotometer (NanoDrop Technologies, Wilmington, DE, USA). One microgram mRNA was used to prepare a library using the Illumina TruSeq ${ }^{\text {TM }}$ RNA Sample Preparation Kit. Paired-end $(2 \times 100$ bp) sequencing was performed on an Illumina HiSeq 2000 (Illumina, Inc., San Diego, CA, USA). Briefly, the mRNA was fragmented, the fragments were copied into first-strand cDNA using reverse transcriptase and random primers, and into second-strand cDNA using DNA Polymerase I and RNase H. Finally, the cDNA was subjected to end repair process.

\section{Data processing and DEG identification}

Total paired-end sequence reads were generated from 22 samples. Before RNA-Seq analysis, the quality of the reads was checked using FastQC v0.11.4 [14]. Next, the reads were trimmed and adapters were removed using SLIDINGWINDOW:4:15 and MINLEN 75 in Trimmomatic-0.38 [15]. The trimmed reads were mapped to the reference genome (Sus_scrofa.Sscrofa11.1.98) using hisat2 v2.1.0 [16]. Subsequently, SAMtools v1.9 was utilized to sort mapped reads and convert data into a binary file format [17]. The sorted binary alignment/map files were quantified using featureCounts (subread-1.6.3-Linux-x86_64) [18]. The trimmed mean of M-values (TMM) normalization [19] was applied to raw counts to estimate relative RNA levels using $\mathrm{R}$ package edgeR [20], and DEGs were identified for each time point $(\mathrm{Days} 3,6,9,12,15$, and 18$)$ relative to Day 0 using thresholds of absolute $\log _{2}$ fold change $(F C) \geq 1$ and false discovery rate $(F D R)<0.05$. To confirm similarities among the samples corresponding to each time point, multidimensional scaling (MDS) was performed using the R package limma [21].

\section{Functional annotation and clustering analysis}

Total DEGs were annotated through the gene ontology (GO) and Kyoto Encyclopedia of Genes and Genomes (KEGG) databases using DAVID v6.8 to identify enriched molecular mechanisms. GO was applied to describe gene products into three independent categories: biological processes, molecular functions, and cellular components. Enriched GO terms were visualized as treemaps using REVIGO [22], and enriched KEGG pathways were illustrated as bar plots with fold enrichment and $P$-values.

DEGs with similar expression patterns, which showed significant expression during at least one of the investigated time points, were clustered by the k-means method with the Pearson correlation distance metric and 1K iterations using Multi-Experiment Viewer (MeV) v4.9.0 [23]. Genes included in each cluster were annotated using the KEGG database, and a network was constructed using ClueGO v2.5.5 with $P$-values $<0.05$ and default values for other options (minimum number of genes $=3$, minimum percentage of genes per term $=4 \%$, and kappa score $=0.4$ ) [24].

\section{Gene Set Enrichment Analysis (GSEA)}

GSEA v4.0.3 based on KEGG was performed to understand the differences in expression of ranked genes at Day 12 relative to Day 0 [25]. In GSEA, TMM normalized counts corresponding to all samples at Days 0 and 12 were used. A normalized enrichment score was calculated by weighted Kolmogorov-Smirnov statistical method using the ranked FCs. To calculate the significance of each KEGG term, a permutation test was performed. The core enriched genes were visualized in a heatmap, and the gene expression modulation for each selected KEGG pathway was illustrated using $\mathrm{R}$ package clusterProfiler [26].

\section{Results}

\section{DEG profiling from the RNA-Seq data}

The RNA-Seq data were generated with an average of 18,978,423 total reads from Day 0 samples, while samples from Days $3,6,9,12,15$, and 18 had averages of 19,492,946, 19,275,046, 18,949,884, 19,281,551, 20,340,490, and 19,497,231 total reads, respectively. Unique mapping rates averaged $94.81 \%$, and overall mapping rates averaged $98.24 \%$ (Table 1). After mapping the reads on the porcine reference genome, similarities among samples were confirmed based on an MDS plot, and each time point was clearly distinguished (Fig. 1B). 
Table 1

Summary of RNA sequencing results and read alignment

\begin{tabular}{|c|c|c|c|c|c|c|c|c|c|c|c|}
\hline \multirow[t]{2}{*}{ No } & \multirow[t]{2}{*}{ Treatment } & \multirow[t]{2}{*}{ ID } & \multicolumn{3}{|l|}{ Raw Data } & \multicolumn{3}{|c|}{ After Trimmomatic } & \multirow{2}{*}{ 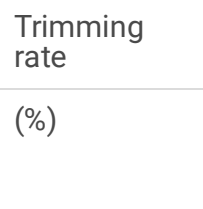 } & \multicolumn{2}{|c|}{ Mapping data } \\
\hline & & & Read & $\begin{array}{l}\text { Sequence } \\
\text { length }\end{array}$ & $\% G C$ & Read & $\begin{array}{l}\text { Sequence } \\
\text { length }\end{array}$ & $\% G C$ & & $\begin{array}{l}\text { Uniquely } \\
\text { mapped } \\
\text { read (\%) }\end{array}$ & $\begin{array}{l}\text { Overall } \\
\text { alignment } \\
\text { rate (\%) }\end{array}$ \\
\hline 1 & Control & $\begin{array}{l}\text { DoC- } \\
\text { Ovid- } \\
1\end{array}$ & $17,961,314$ & 101 & 51 & $15,342,621$ & $75-101$ & 50 & 14.57962931 & 94.85 & 98.29 \\
\hline 2 & & $\begin{array}{l}\text { DoC- } \\
\text { Ovid- } \\
2\end{array}$ & $19,106,487$ & 101 & 51 & $16,281,159$ & $75-101$ & 50 & 14.78727094 & 94.82 & 98.33 \\
\hline 3 & & $\begin{array}{l}\text { D0C- } \\
\text { Ovid- } \\
3\end{array}$ & $19,867,469$ & 101 & 51 & $17,014,555$ & $75-101$ & 50 & 14.35972544 & 94.86 & 98.37 \\
\hline 4 & Treat & $\begin{array}{l}\text { D3C- } \\
\text { Ovid- } \\
1\end{array}$ & $19,772,368$ & 101 & 51 & $16,953,808$ & $75-101$ & 50 & 14.25504522 & 94.94 & 98.34 \\
\hline 5 & & $\begin{array}{l}\text { D3C- } \\
\text { Ovid- } \\
2\end{array}$ & $19,530,742$ & 101 & 51 & $16,828,423$ & $75-101$ & 50 & 13.83623315 & 94.92 & 98.39 \\
\hline 6 & & $\begin{array}{l}\text { D3C- } \\
\text { Ovid- } \\
3\end{array}$ & $19,175,729$ & 101 & 50 & $16,371,028$ & $75-101$ & 49 & 14.62630704 & 94.40 & 98.05 \\
\hline 7 & Treat & $\begin{array}{l}\text { D6C- } \\
\text { Ovid- } \\
2\end{array}$ & $18,510,823$ & 101 & 50 & $15,731,900$ & $75-101$ & 49 & 15.01242273 & 94.97 & 98.21 \\
\hline 8 & & $\begin{array}{l}\text { D6C- } \\
\text { Ovid- } \\
3\end{array}$ & $20,039,268$ & 101 & 50 & $17,036,473$ & $75-101$ & 49 & 14.98455433 & 95.04 & 98.21 \\
\hline 9 & Treat & $\begin{array}{l}\text { D9C- } \\
\text { Ovid- } \\
1\end{array}$ & $18,665,067$ & 101 & 50 & $16,000,684$ & $75-101$ & 49 & 14.27470365 & 94.82 & 98.19 \\
\hline 10 & & $\begin{array}{l}\text { D9C- } \\
\text { Ovid- } \\
2\end{array}$ & $18,732,721$ & 101 & 50 & $16,011,408$ & 75-101 & 49 & 14.52705669 & 94.70 & 98.11 \\
\hline 11 & & $\begin{array}{l}\text { D9C- } \\
\text { Ovid- } \\
3\end{array}$ & $19,451,864$ & 101 & 51 & $16,599,341$ & 75-101 & 50 & 14.66452264 & 94.63 & 98.20 \\
\hline 12 & Treat & $\begin{array}{l}\text { D12C- } \\
\text { Ovid- } \\
1\end{array}$ & $19,885,880$ & 101 & 50 & $17,038,736$ & $75-101$ & 50 & 14.31741517 & 94.81 & 98.25 \\
\hline 13 & & $\begin{array}{l}\text { D12C- } \\
\text { Ovid- } \\
2\end{array}$ & $17,698,398$ & 101 & 50 & $15,111,105$ & 75-101 & 49 & 14.6187977 & 94.79 & 98.19 \\
\hline 14 & & $\begin{array}{l}\text { D12C- } \\
\text { Ovid- } \\
3\end{array}$ & $18,994,386$ & 101 & 50 & $16,222,068$ & 75-101 & 49 & 14.59545994 & 94.87 & 98.20 \\
\hline 15 & & $\begin{array}{l}\text { D12C- } \\
\text { Ovid- } \\
4\end{array}$ & $20,547,540$ & 101 & 50 & $17,823,244$ & 75-101 & 49 & 13.25850199 & 94.76 & 98.12 \\
\hline 16 & Treat & $\begin{array}{l}\text { D15C- } \\
\text { Ovid- } \\
1\end{array}$ & $21,071,137$ & 101 & 51 & $18,332,506$ & 75-101 & 50 & 12.99707273 & 94.35 & 98.13 \\
\hline 17 & & $\begin{array}{l}\text { D15C- } \\
\text { Ovid- } \\
2\end{array}$ & $21,085,713$ & 101 & 50 & $18,321,567$ & 75-101 & 49 & 13.1090943 & 94.70 & 98.10 \\
\hline 18 & & $\begin{array}{l}\text { D15C- } \\
\text { Ovid- } \\
3\end{array}$ & $20,769,207$ & 101 & 51 & $17,992,010$ & $75-101$ & 50 & 13.37170456 & 94.84 & 98.20 \\
\hline
\end{tabular}




\begin{tabular}{|c|c|c|c|c|c|c|c|c|c|c|c|}
\hline 19 & & $\begin{array}{l}\text { D15C- } \\
\text { Ovid- } \\
4\end{array}$ & $18,435,904$ & 101 & 51 & $15,916,002$ & 75-101 & 50 & 13.66844826 & 94.67 & 98.23 \\
\hline 20 & Treat & $\begin{array}{l}\text { D18C- } \\
\text { Ovid- } \\
1\end{array}$ & $20,404,927$ & 101 & 50 & $17,614,747$ & 75-101 & 50 & 13.67405039 & 94.78 & 98.20 \\
\hline 21 & & $\begin{array}{l}\text { D18C- } \\
\text { Ovid- } \\
2\end{array}$ & $19,555,704$ & 101 & 50 & $16,979,038$ & 75-101 & 49 & 13.17603294 & 95.33 & 98.58 \\
\hline 22 & & $\begin{array}{l}\text { D18C- } \\
\text { Ovid- } \\
3\end{array}$ & $18,531,061$ & 101 & 50 & $16,045,886$ & 75-101 & 50 & 13.4108619 & 94.96 & 98.35 \\
\hline
\end{tabular}

Our results showed that the number of DEGs gradually increased following Days $3(n=641), 6(n=1,450)$, and $9(n=1,879)$. The number of DEGs peaked on Day $12(n=2,343)$ and then decreased steadily from Day $15(n=932)$ to Day $18(n=423)$. The numbers of DEGs with upregulated and downregulated expressions compared to the Day 0 also showed the same trend as the total number of DEGs. Moreover, the number of DEGs $(n=4,931)$ with downregulated expression was consistently greater than of DEGs with upregulated expression $(n=2,692)$ (Fig. 1C and Additional file 1).

\section{Functional annotations}

GO enrichment analyses were performed to identify the biological processes of DEGs at each time point, and the results are displayed in treemaps indicating $\log _{10} P$-value as an area (Fig. 2). The results showed that $\mathrm{GO}$ terms were enriched in the negative regulation of osteoblast differentiation on Day 3 and significantly enriched in the positive regulation of cell proliferation on Days 6, 9, and 12. On Day 15, collagen fibril organization was enriched, while on Day 18 , the chemokine-mediated signalling pathway was enriched.

At each time point, enriched pathways for DEGs were determined using the KEGG database (Fig. 3 and Additional file 2). Cell cycle, adrenergic signalling in cardiomyocytes, and p53 signalling pathway were enriched on Day 3. On Day 6, protein digestion and absorption, focal adhesion, and PI3K-Akt signalling pathway were enriched. Calcium signalling pathway, neuroactive ligand-receptor interaction, and proteoglycans in cancer were enriched on Days 9 and 12. Genes sampled on Day 15 were associated with enrichment in neuroactive ligand-receptor interaction, complement and coagulation cascades, and extracellular matrix (ECM)-receptor interaction. On Day 18, cell cycle, DNA replication, and HTLV-I infection were significantly enriched.

Notably, analyses of gene expression on Days 6, 9, and 12 (the luteal phase of the oestrous cycle) revealed five common, significantly enriched pathways (calcium signalling pathway, ECM-receptor interaction, focal adhesion, oocyte meiosis, and PI3K-Akt signalling pathway), and these days were closely grouped in the MDS plot (Fig. 1B) and had highly increased numbers of DEGs (Fig. 1C).

\section{Clustering by gene expression pattern}

To examine the expression patterns of DEGs at different time points of the oestrous cycle, the entire set of identified DEGs was adopted for kmeans clustering analysis and sorted into three major clusters. Next, we applied network analysis based on the KEGG database using the ClueGO plugin to determine the functions of the clustered genes. Clusters 1 and 2 consisted of 1,222 and 1,146 genes, respectively, which showed contrasting expression patterns. The expression of genes in Cluster 1 was upregulated throughout the oestrous cycle, while the expressions of genes in Cluster 2 was downregulated (Fig. 4A and 4B). A network was constructed with genes from Clusters 1 and 2 involved in any of the five significant KEGG pathways (Fig. 4C). The genes in Cluster 1 were primarily involved in the PI3K-Akt signalling and steroid hormone biosynthesis pathways, while the genes in Cluster 2 were involved in the ECM-receptor interaction and protein digestion and absorption pathways. Figure 5 shows that the Cluster 3 showed a clearly different pattern of gene expression compared to Clusters 1 and 2 . The pattern in Cluster 3 showed uniquely downregulated genes on Day 12 (Fig. 5A). Subsequently, we constructed a network from 1,000 genes of Cluster 3 associated with six significant KEGG pathway terms (cell cycle, compartment and coagulation cascades, calcium signalling, insulin secretion, oocyte meiosis, and dilated cardiomyopathy; Fig. 5B). Among these pathways, those involved in calcium signalling and oocyte meiosis were not only validated for their high activity on Day 12 referring to the KEGG pathways (Fig. 5B), but were also significantly represented in the GO and KEGG results of the luteal phase, which validated their significance (Figs. 1 and 2).

\section{Gene Set Enrichment Analysis (GSEA)}

Further analysis was focused on the transcriptomic changes on Day 12 in Cluster 3, which showed a uniquely and significantly downregulated expression profile during the oestrous cycle. In GSEA, calcium signalling pathway and oocyte meiosis were highly ranked terms (Fig. 6A): the heatmaps showed 61 core enriched genes in calcium signalling pathway and 37 in oocyte meiosis (Fig. 6B and 6C). The modulation in expression of genes related to the two pathways indicated they were mostly downregulated (Fig. 6D and 6E). 


\section{Discussion}

DEGs at different time points of the oestrous cycle in the oviduct during the luteal phase

During the oestrous cycle, ovarian steroid hormones, including oestrogen and progesterone, affect oviductal, morphological, and functional responses [2]. Thus, it is important to understand the changes in molecular mechanisms and functions of the oviduct during the oestrous cycle. In this study, the molecular mechanisms regulating dynamic changes in the transcriptome at different time points of the oestrous cycle were identified. As shown in the MDS plot, during the luteal phase (Days 6, 9, 12), samples were grouped together by transcriptome expression on each day and showed similar expression patterns (Fig. 1B). The numbers of DEGs on Days 6, 9, and 12 were 1,405, 1,879, and 2,343, respectively, indicating that the number of significant DEGs gradually increased during luteal phase. Among KEGG pathways enriched in the luteal phase (Fig. 3), calcium signalling pathway plays an important role in normal oocyte development in the oviduct [27], while ECM-receptor interaction plays a role in protecting and supporting cells during ovulation [28, 29]. Focal adhesions are involved in receptor binding to the ECM, which is crucial for oocyte fertilization [10]. Oocyte meiosis is regulated by progesterone, which promotes oocyte development until fertilization. The PI3K-Akt signalling pathway regulates cell processes such as metabolism, proliferation, and cell survival [30].

\section{Cell-to-cell interaction and proliferation in the oviduct}

Using k-means clustering, we selected three clusters with similar gene expression patterns based on the KEGG pathway database (Fig. 3). The overall physiological changes through the course of oestrous cycle were identified by integrating and analysing Clusters 1 and 2 . In particular, the significantly enriched pathways, including ECM-receptor interaction, PI3K-Akt signalling, protein digestion and absorption, glycosaminoglycan biosynthesis, and proteoglycans in cancer, were remarkably associated with oviductal activities during the oestrous cycle. The PI3K-Akt signalling pathway is related to cell physiology and proliferation [31] and stimulates follicle development and oocyte growth [30]. The ECM plays an essential role in embryo development and regulates different cellular processes via expression of ECM proteins [32, 33]. Moreover, interactions between the cumulus-oocyte complex and epithelial cells of the oviduct are regulated at the level of the ECM [34]. Through its complexes of elastin, proteoglycans, and glycosaminoglycans, the ECM also causes uterus dilation through collagen fibre degradation during the oestrous cycle [35]. Overall, our results revealed significant roles played by time-dependent transcriptomic changes in cell growth, proliferation, and cell-cell interaction during the oestrous cycle.

\section{Oocyte meiosis and calcium signalling pathway}

The expression levels of Cluster 3 genes gradually increased over the luteal phase and rapidly decreased after Day 12 (Fig. 5A). Moreover, oocyte meiosis and calcium signalling pathway were most significantly enriched in Cluster 3 genes on Day 12 . Furthermore, similar results appeared in the top terms when listed in an order of significance based on the pathways activated on Day 12 and identified via GSEA (Fig. $6 \mathrm{~A}$ ).

Oocyte meiosis is related to the formation and development of oocytes and is an important process involved in oocyte migration and fertilization, the core functions of the oviduct [36]. IGF1, PGR, ITPR3, CAMK2A, CAMK2B, IP3R, BUB1, and CDC20 were downregulated in the enriched KEGG pathways (Fig. 6B and 6D). Previously, IGF1, PGR, ITPR3, CAMK2B, and PLK1 were shown to be involved in oocyte-related processes. IGF1 plays as an important role during early oestrous cycle, and ovulation fails if IGF1 is not expressed [37]. In vivo, IGF1 may indirectly induce the stimulation of cumulus and/or granulosa cells, resulting in improved oocyte maturation and fertilization [38]. $P G R$ is a progesterone receptor that mediates the effects of progesterone in the oviduct. It plays an important role in the successful release of oocytes from preovulatory follicles [39] as well as in ciliary beat frequency and oocyte transport [2]. ITPR3 is involved in the initiation and propagation of intracellular $\mathrm{Ca}^{2+}$ signalling during the follicular phase [40], while CAMK2B and PLK1 encode essential factors involved in Ca ${ }^{2+}$-induced exit from mitosis [41]. Hence, these genes are involved in successful ovulation and fertilization. Therefore, based on the predominance of downregulated genes on Day 12, we suspect that oestrous cycle-related activities take place at a site other than oviduct and active activities occur in follicular phase, where oocytes present in the oviduct.

KEGG pathway analysis identified calcium signalling as the most enriched pathway. Progesterone, an ovarian steroid hormone secreted in the oviduct, activates calcium channels and increases intracellular calcium levels in sperm to promote their hyperactivity [42]. Reportedly, sperm hyperactivity assists in escaping epithelial folds, allowing sperm to reach oocytes [43]. Among the significant DEGs identified by GSEA, ITPR1 and $C A M K 1 D$ are involved in calcium signalling pathways and associated with fertilization (Fig. 6C and 6E), ITPR1 modulates intracellular calcium ion concentrations during mammalian fertilization [44]; CAMK1D also serves as a mediator of calcium-dependent cellular processes [45]. We speculate that the predominance of downregulated genes during the luteal phase (Day 12) may indicate their roles being less relevant to fertilization.

As mentioned earlier, oocyte transport is an essential function of the oviduct. In the oviduct, oestrogen and progesterone regulate oocyte transport, cilia beating, and smooth muscle contraction [46]. Cluster profiling revealed a gene involved in calcium signalling pathway that plays a role in the smooth muscle contraction in the oviduct (Fig. 6E). DEGs related to oocyte transport were identified by functional analysis and 
GSEA: activation of $C H R M 3$ is associated with muscle contraction as the $\mathrm{M}_{3}$ receptor mediates intracellular calcium [47], while TNNC1 is involved in calcium contractile events by binding with $\mathrm{Ca}^{2+}$ [48]. Downregulation of these genes on Day 12 and observation of smooth muscle relaxation explains why muscle contraction is inhibited in the luteal phase.

\section{Conclusion}

This study provides novel insights in dynamic changes of molecular mechanisms in oviducts of non-fertilization gilts during oestrous cycle through RNA-Seq technology. In the oviduct, genes associated with oocyte meiosis and calcium signalling pathways are significantly upregulated during the oestrous cycle as their expression influences oocyte transport and fertilization, the core functions of the oviduct. Current result led us to convince the evidence that oocyte maturation and smooth muscle contraction are regulated by IGF1, PGR, ITPR1, and CHRM3. Hence, this study could become a valuable resource for further study of porcine reproductive tissue as a basis for successful reproduction and breeding in the pig industry and uncovers an overall mechanism change for the pig oviduct in the oestrous cycle. We expect future studies to further clarify the regulatory roles of genes that are differently expressed in the follicular and luteal phases.

\section{Abbreviations}

$\mathrm{CL}$, corpus luteum

DEGs, differentially expressed genes

ECM, extracellular matrix

FC, fold change

FDR, false discovery rate

GO, gene ontology

GSEA, Gene Set Enrichment Analysis

KEGG, Kyoto Encyclopedia of Genes and Genomes

LH, luteinizing hormone

MDS, multidimensional scaling

MeV, Multi-Experiment Viewer

RNA-Seq, RNA sequencing

TMM, trimmed mean of M-values

\section{Declarations}

\section{Ethics approval}

All animal-related experimental procedures were performed in accordance with the Guide for Care and Use of Animals in Research and approved by the Institutional Animal Care and Use Committee of the National Institute of Animal Science (No. 2015-137).

\section{Consent for publication}

Not applicable.

\section{Availability of data and material}

The datasets generated and/or analysed during the current study are available in the GSE108570 repository, NCBI Gene Expression Omnibus (https://www.ncbi.nlm.nih.gov/geo/).

\section{Competing interests}

The authors declare that they have no competing interests. 


\section{Funding}

This research was supported by the Basic Science Research Program through the National Research Foundation of Korea (NRF), funded by the Ministry of Education (NRF-2018R1A6A1A03025159). The funding bodies had no role in the design of the study; the collection, analysis, and interpretation of the data; or the writing of the manuscript.

\section{Authors' contributions}

MJJ analysed the data and wrote the manuscript; CL assisted with data analysis; BL and JMK reviewed and edited the manuscript. All authors have read and agreed to the published version of the manuscript.

\section{Acknowledgments}

This research was supported by the Chung-Ang University Graduate Research Scholarship in 2021.

\section{References}

1. Irvin KM, Swiger LA. Genetic and phenotypic parameters for sow productivity. J Anim Sci. 1984;58(5):1144-50.

2. Akison LK, Robker RL. The critical roles of progesterone receptor (PGR) in ovulation, oocyte developmental competence and oviductal transport in mammalian reproduction. Reprod Domest Anim. 2012;47(Suppl 4):288-96.

3. Henricks DM, Guthrie HD, Handlin DL. Plasma estrogen, progesterone and luteinizing hormone levels during the estrous cycle in pigs. Biol Reprod. 1972;6(2):210-8.

4. Soede NM, Langendijk P, Kemp B. Reproductive cycles in pigs. Anim Reprod Sci. 2011;124(3-4):251-8.

5. Younis A, Brackett B, Fayrer-Hosken R. Influence of serum and hormones on bovine oocyte maturation and fertilization in vitro. Gamete research. 1989;23(2):189-201.

6. Acuna OS, Aviles M, Lopez-Ubeda R, Guillen-Martinez A, Soriano-Ubeda C, Torrecillas A, et al. Differential gene expression in porcine oviduct during the oestrous cycle. Reprod Fertil Dev. 2017;29(12):2387-99.

7. Park ST, Kim J. Trends in Next-Generation Sequencing and a New Era for Whole Genome Sequencing. Int Neurourol J. 2016;20(Suppl 2):76-83.

8. Wang Z, Gerstein M, Snyder M. RNA-Seq: a revolutionary tool for transcriptomics. Nat Rev Genet. 2009;10(1):57-63.

9. Zhao S, Fung-Leung WP, Bittner A, Ngo K, Liu X. Comparison of RNA-Seq and microarray in transcriptome profiling of activated T cells. PLoS One. 2014;9(1):e78644.

10. Kim JM, Park JE, Yoo I, Han J, Kim N, Lim WJ, et al. Integrated transcriptomes throughout swine oestrous cycle reveal dynamic changes in reproductive tissues interacting networks. Sci Rep. 2018;8(1):5436.

11. Fischer D, Laiho A, Gyenesei A, Sironen A. Identification of reproduction-related gene polymorphisms using whole transcriptome sequencing in the Large White pig population. G3: Genes, Genomes, Genetics. 2015;5(7):1351-60.

12. Geisert RD, Renegar RH, Thatcher WW, Roberts RM, Bazer FW. Establishment of pregnancy in the pig: I. Interrelationships between preimplantation development of the pig blastocyst and uterine endometrial secretions. Biol Reprod. 1982;27(4):925-39.

13. Simms D, Cizdziel PE, Chomczynski P. TRIzol. A new reagent for optimal single-step isolation of RNA. Focus. 1993;15(4):532-5.

14. Andrews S. FastQC: a quality control tool for high throughput sequence data. Cambridge: Babraham Bioinformatics, Babraham Institute; 2010.

15. Williams CR, Baccarella A, Parrish JZ, Kim CC. Trimming of sequence reads alters RNA-Seq gene expression estimates. BMC Bioinform. 2016;17(1):103.

16. Kim D, Langmead B, Salzberg SL. HISAT: a fast spliced aligner with low memory requirements. Nature methods. 2015;12(4):357-60.

17. Li H, Handsaker B, Wysoker A, Fennell T, Ruan J, Homer N, et al. The sequence alignment/map format and SAMtools. Bioinformatics. 2009;25(16):2078-9.

18. Liao Y, Smyth GK, Shi W. featureCounts: an efficient general purpose program for assigning sequence reads to genomic features. Bioinformatics. 2014;30(7):923-30.

19. Robinson MD, Oshlack A. A scaling normalization method for differential expression analysis of RNA-seq data. Genome biology. 2010;11(3):R25.

20. Robinson MD, McCarthy DJ, Smyth GK. edgeR: a Bioconductor package for differential expression analysis of digital gene expression data. Bioinformatics. 2010;26(1):139-40. 
21. Ritchie ME, Phipson B, Wu D, Hu Y, Law CW, Shi W, et al. limma powers differential expression analyses for RNA-sequencing and microarray studies. Nucleic acids research. 2015;43(7):e47-e.

22. Supek F, Bošnjak M, Škunca N, Šmuc T. REVIGO summarizes and visualizes long lists of gene ontology terms. PloS one. 2011;6(7).

23. Howe EA, Sinha R, Schlauch D, Quackenbush J. RNA-Seq analysis in MeV. Bioinformatics. 2011;27(22):3209-10.

24. Bindea G, Mlecnik B, Hackl H, Charoentong P, Tosolini M, Kirilovsky A, et al. ClueGO: a Cytoscape plug-in to decipher functionally grouped gene ontology and pathway annotation networks. Bioinformatics. 2009;25(8):1091-3.

25. Subramanian A, Tamayo P, Mootha VK, Mukherjee S, Ebert BL, Gillette MA, et al. Gene set enrichment analysis: a knowledge-based approach for interpreting genome-wide expression profiles. Proceedings of the National Academy of Sciences. 2005;102(43):15545-50.

26. Yu G, Wang L-G, Han Y, He Q-Y. clusterProfiler: an R package for comparing biological themes among gene clusters. Omics: a journal of integrative biology. 2012;16(5):284-7.

27. Stricker SA. Comparative biology of calcium signaling during fertilization and egg activation in animals. Dev Biol. 1999;211(2):157-76.

28. Russell DL, Salustri A. Extracellular matrix of the cumulus-oocyte complex. Semin Reprod Med. 2006;24(4):217-27.

29. Bazer FW, Song G, Kim J, Dunlap KA, Satterfield MC, Johnson GA, et al. Uterine biology in pigs and sheep. J Anim Sci Biotechnol. 2012;3(1):23.

30. Andrade GM, da Silveira JC, Perrini C, Del Collado M, Gebremedhn S, Tesfaye D, et al. The role of the PI3K-Akt signaling pathway in the developmental competence of bovine oocytes. PLoS One. 2017;12(9):e0185045.

31. Dobbin ZC, Landen CN. The importance of the PI3K/AKT/MTOR pathway in the progression of ovarian cancer. Int J Mol Sci. 2013;14(4):8213-27.

32. Juliano RL, Haskill S. Signal transduction from the extracellular matrix. J Cell Biol. 1993;120(3):577-85.

33. Roskelley CD, Srebrow A, Bissell MJ. A hierarchy of ECM-mediated signalling regulates tissue-specific gene expression. Curr Opin Cell Biol. 1995;7(5):736-47.

34. Ulbrich SE, Schoenfelder M, Thoene S, Einspanier R. Hyaluronan in the bovine oviduct-modulation of synthases and receptors during the estrous cycle. Mol Cell Endocrinol. 2004;214(1-2):9-18.

35. Rodriguez-Pinon M, Tasende C, Casuriaga D, Bielli A, Genovese P, Garofalo EG. Collagen and matrix metalloproteinase-2 and - 9 in the ewe cervix during the estrous cycle. Theriogenology. 2015;84(5):818-26.

36. Luvoni GC, Chigioni S, Allievi E, Macis D. Meiosis resumption of canine oocytes cultured in the isolated oviduct. Reprod Domest Anim. 2003;38(5):410-4.

37. Velazquez MA, Zaraza J, Oropeza A, Webb R, Niemann H. The role of IGF1 in the in vivo production of bovine embryos from superovulated donors. Reproduction. 2009;137(2):161-80.

38. Xia P, Tekpetey FR, Armstrong DT. Effect of IGF-I on pig oocyte maturation, fertilization, and early embryonic development in vitro, and on granulosa and cumulus cell biosynthetic activity. Mol Reprod Dev. 1994;38(4):373-9.

39. Kim J, Bagchi IC, Bagchi MK. Control of ovulation in mice by progesterone receptor-regulated gene networks. Mol Hum Reprod. 2009;15(12):821-8.

40. Mikoshiba K. The IP3 receptor/Ca2 + channel and its cellular function. Biochem Soc Symp. 2007(74):9-22.

41. Hansen DV, Tung JJ, Jackson PK. CaMKII and polo-like kinase 1 sequentially phosphorylate the cytostatic factor Emi2/XErp1 to trigger its destruction and meiotic exit. Proc Natl Acad Sci U S A. 2006;103(3):608-13.

42. Coy P, Garcia-Vazquez FA, Visconti PE, Aviles M. Roles of the oviduct in mammalian fertilization. Reproduction. 2012;144(6):649-60.

43. Demott RP, Suarez SS. Hyperactivated sperm progress in the mouse oviduct. Biol Reprod. 1992;46(5):779-85.

44. Malcuit C, Knott JG, He C, Wainwright T, Parys JB, Robl JM, et al. Fertilization and inositol 1,4,5-trisphosphate (IP3)-induced calcium release in type-1 inositol 1,4,5-trisphosphate receptor down-regulated bovine eggs. Biol Reprod. 2005;73(1):2-13.

45. Braun AP, Schulman H. The multifunctional calcium/calmodulin-dependent protein kinase: from form to function. Annu Rev Physiol. 1995;57:417-45.

46. Mizobe Y, Yoshida M, Miyoshi K. Enhancement of cytoplasmic maturation of in vitro-matured pig oocytes by mechanical vibration. Journal of Reproduction and Development. 2010:1001220244-.

47. Yang CM, Yo YL, Wang YY. Intracellular calcium in canine cultured tracheal smooth muscle cells is regulated by M3 muscarinic receptors. Br J Pharmacol. 1993;110(3):983-8.

48. Herzberg O, Moult J, James MN. A model for the Ca2+-induced conformational transition of troponin C. A trigger for muscle contraction. J Biol Chem. 1986;261(6):2638-44.

\section{Figures}

Page 9/15 
A

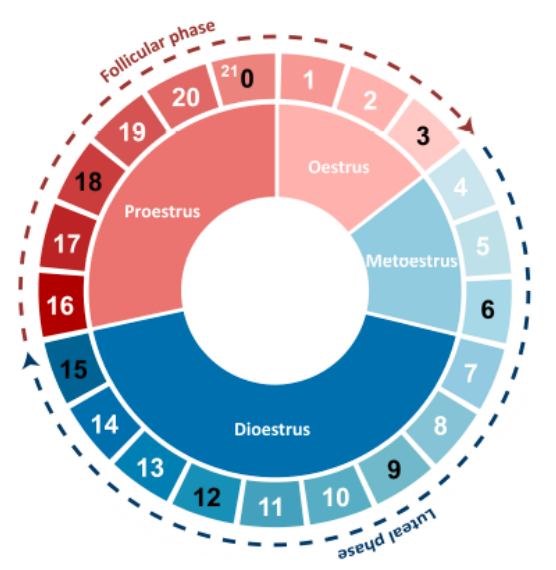

B

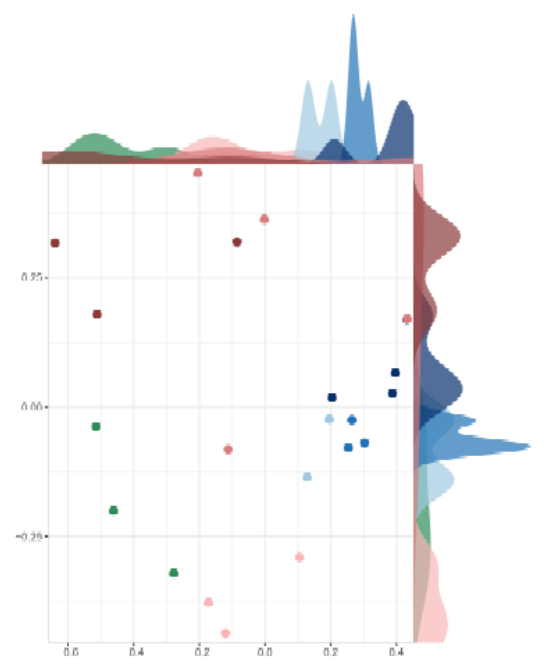

C

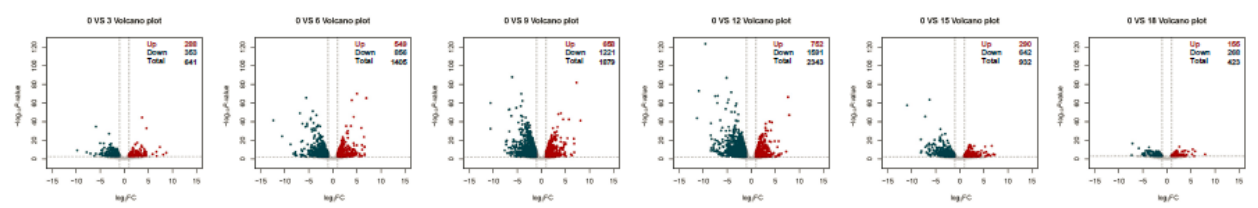

1200

800

400

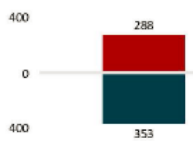

800

1200

1600

Number of differentially expressed genes (DEGs)

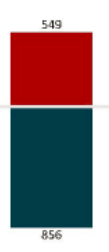

ovs 6

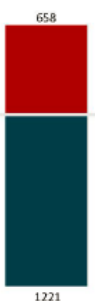

ovs 9

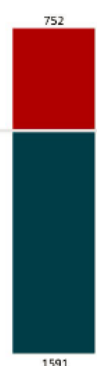

ovs 12

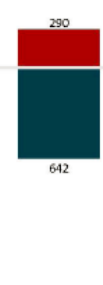

ovs 15

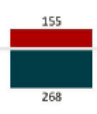

ovs 18

\section{Figure 1}

Oviduct transcriptome analysis during the swine oestrous cycle. (A) Oviduct samples at time points (Day 0; D00, Day 3; D03, Day 6; D06, Day 9; D09, Day 12; D12, Day 15; D15, and Day 18; D18) during the oestrous cycle. (B) Relationship between different time point samples by multidimensional scaling (MDS) analysis. Each point represents an oviduct sample. (C) Dynamic view of a volcano plot of DEGs (FDR $<0.05$ and $\log 2 \mathrm{FC} \geq 1$ for upregulated and log2 FC $\leq-1$ for downregulated expression) at Days $3,6,9,12,15$, and 18 relative to expression at Day 0 . DEGs with upregulated expression are show in red, and those with downregulated expression are shown in blue. The $X$ and $Y$ axes were scaled to log FC and - log10 P-value. The bar graph shows the number of DEGs at each time point. 
A
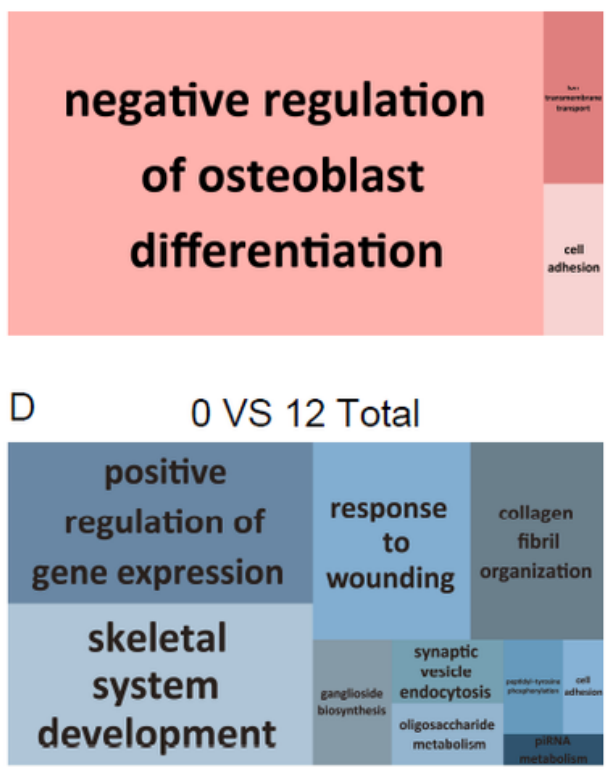

B

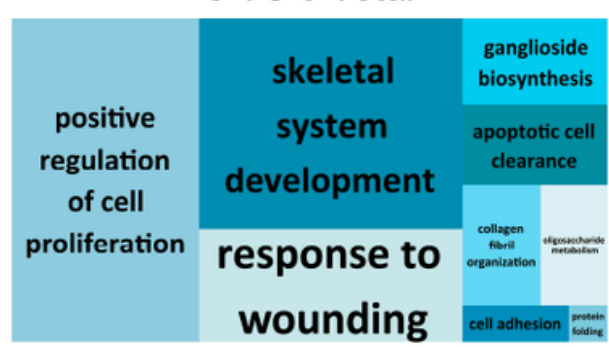

$\mathrm{E}$

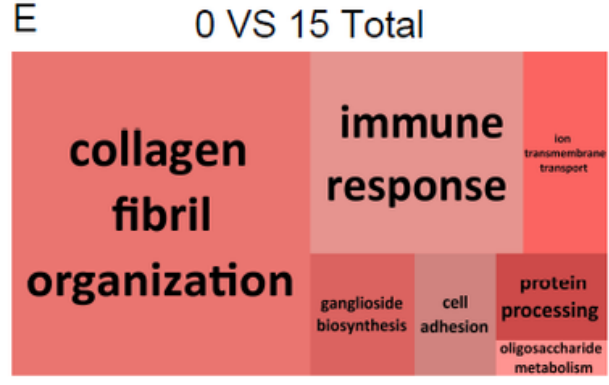

C

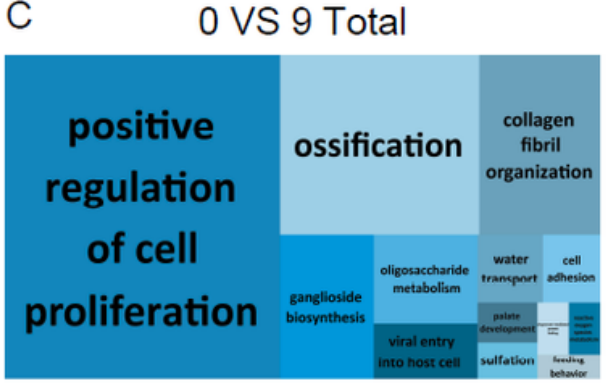

$\mathrm{F}$

0 VS 18 Total

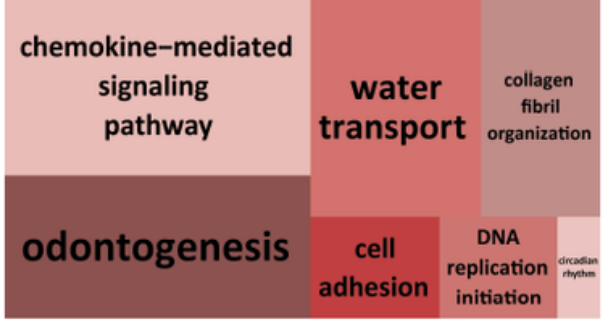

Figure 2

Gene Ontology treemaps of biological process terms according to time points using differentially expressed genes from databases in DAVID. Treemaps were constructed based on P-values. (A-F) 
A

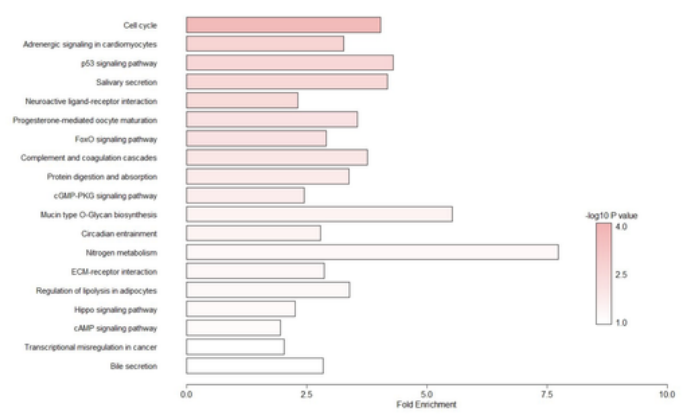

C

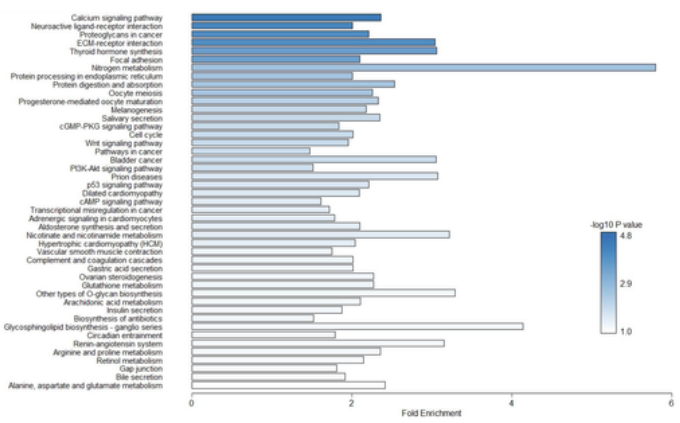

$\mathrm{E}$

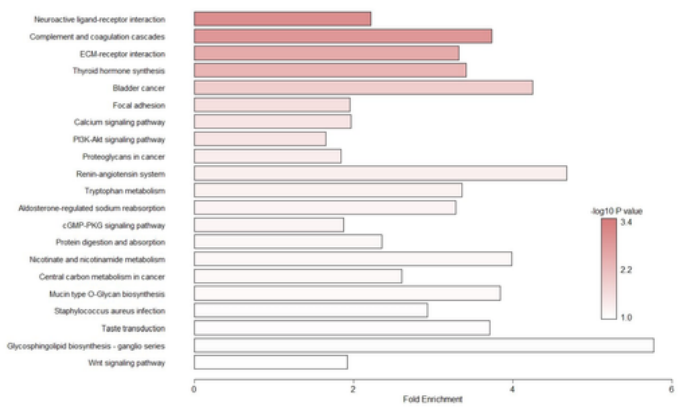

B

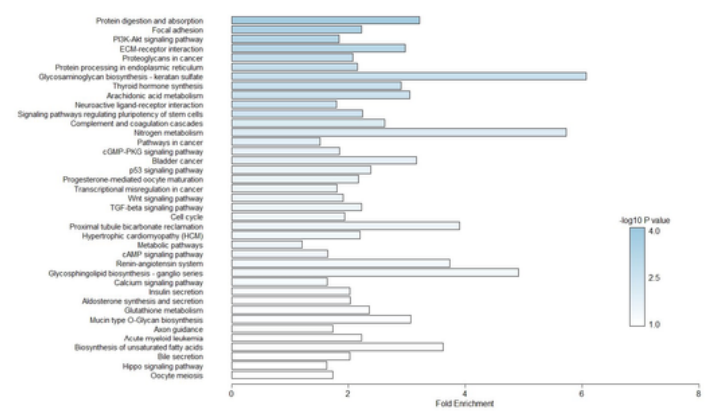

D

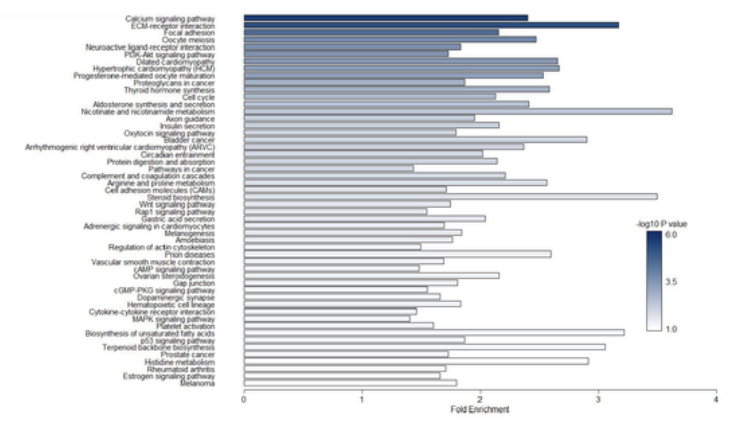

$\mathrm{F}$

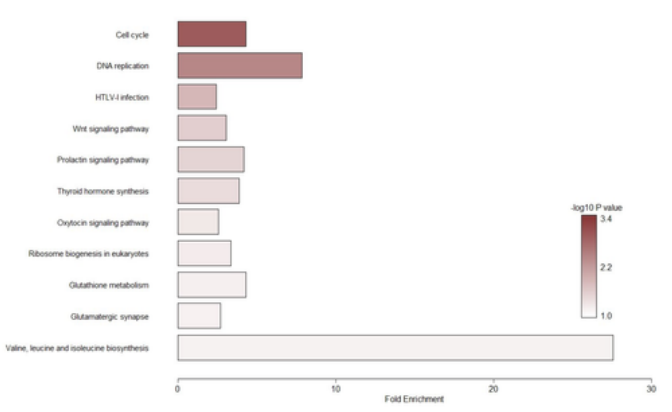

Figure 3

Enrichment analysis of differentially expressed genes in KEGG pathways from databases in DAVID. Enriched pathways for the time points were applied in order to fold change and - log10P-value. Each graph shows the results in order of progressing oestrous cycle. (A; Day 3, B; Day 6, C; Day 9, D; Day 12, E; Day 15, F; Day 18) 
A 2

1.5

1

0.5

0

$-0.5$

$-1$

$-1.5$

$-2$

Ovs3

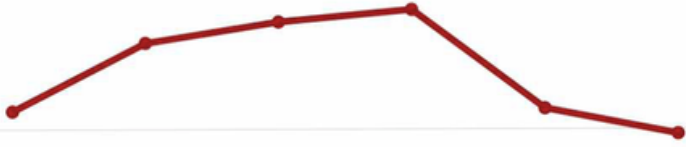

B 2

1.5

1

0.5

0

$-0.5$

$-1$

$-1.5$

$-2$

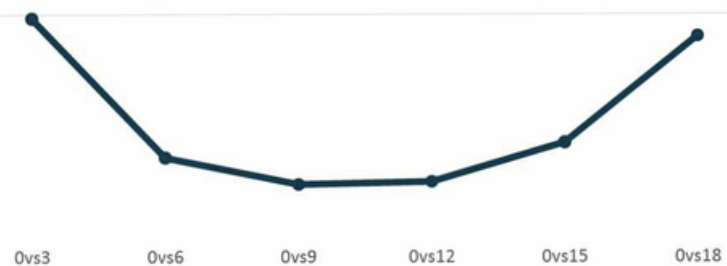

C

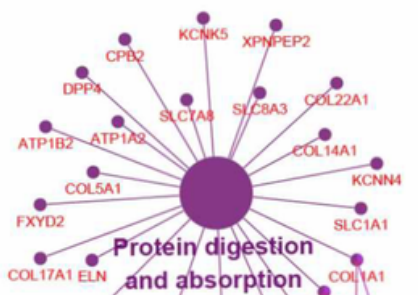

COL_3A1
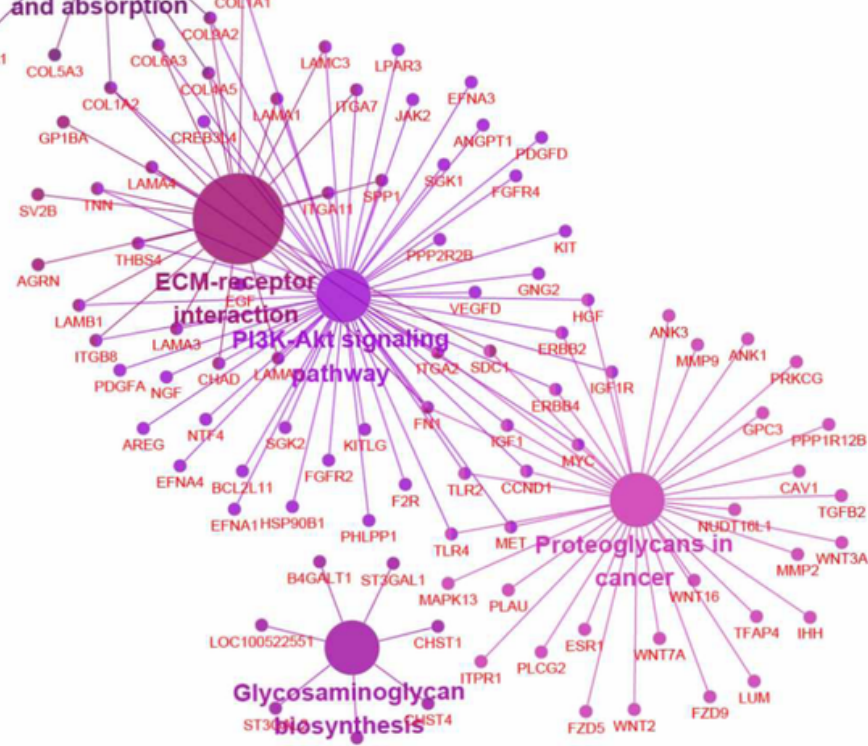

Ovs9

Ovs12

Ovs18

\section{Figure 4}

DEGs were sorted by MeV Clustering. (A) Cluster 1 contained 1,222 DEGs (gene expression pattern in red). (B) DEGs with upregulated expression are show in red, and those with downregulated expression are shown in blue. Cluster 2 contained 1,146 DEGs (gene expression pattern in blue). Overall gene expression patterns for Clusters 1 and 2 was similar, but Cluster 1 showed an upregulated trend, while Cluster 2 showed a downregulated trend. (C) ClueGO (v. 2.5.5; P-value $\leq 0.05$, kappa score $=0.4$ ) plugin was used with the KEGG database to visualize genes related to each pathway. 
A

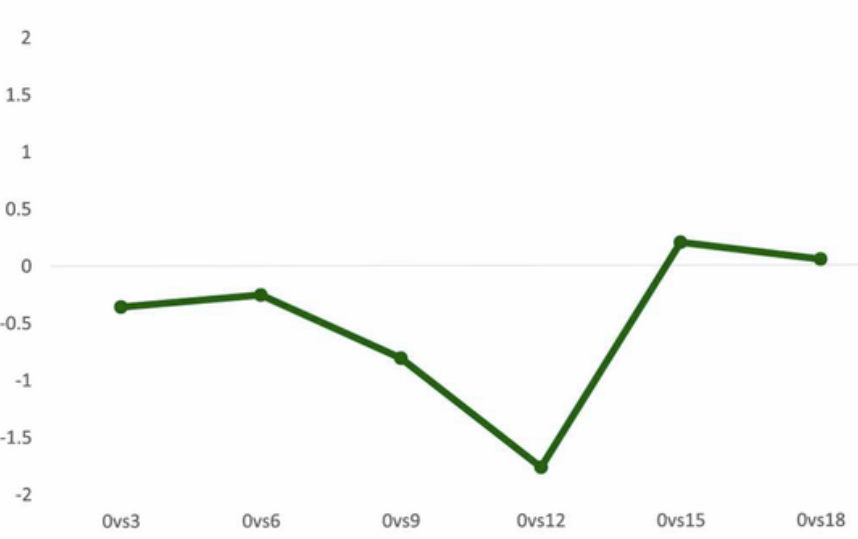

B

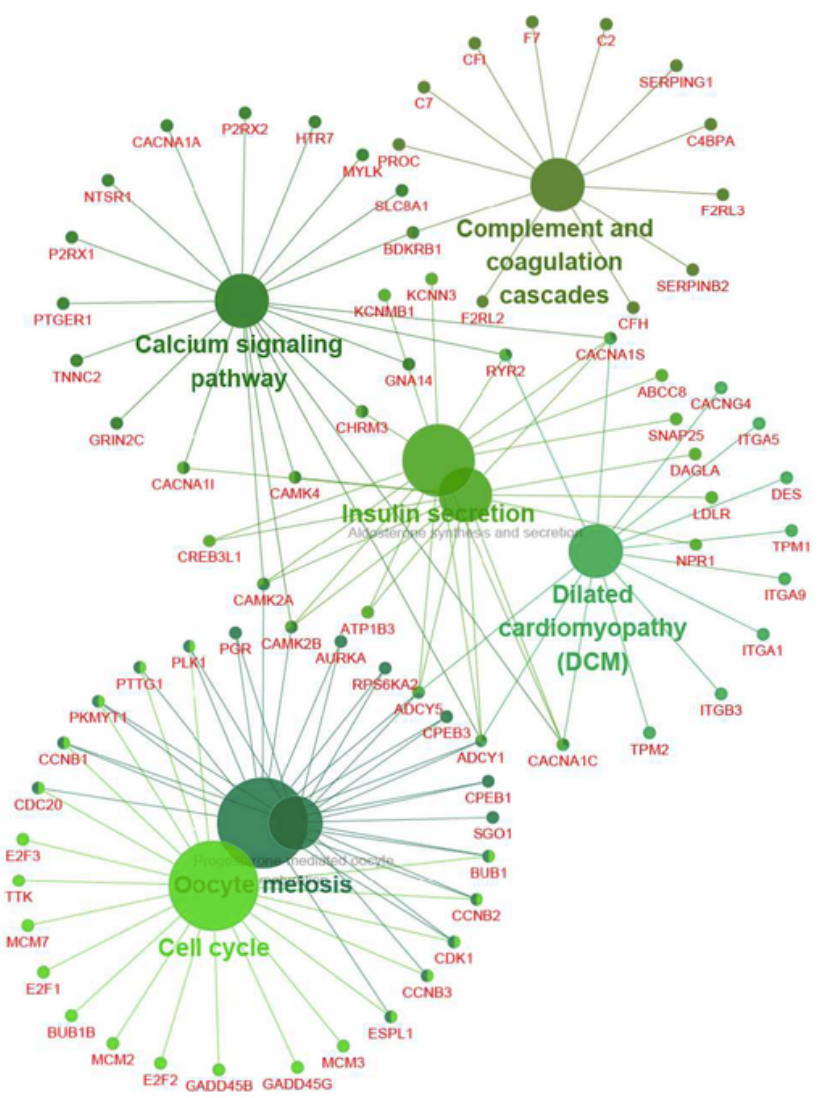

Figure 5

DEGs were sorted by MeV Clustering. (A) Cluster 3 contained 1,000 DEGs (gene expression pattern in green) and showed a downregulated expression trend on Day 12. (B) ClueGO (v. 2.5.5, P-value $\leq 0.05$, kappa score $=0.4)$ plugin was used with the KEGG database to visualize genes related to each pathway. 


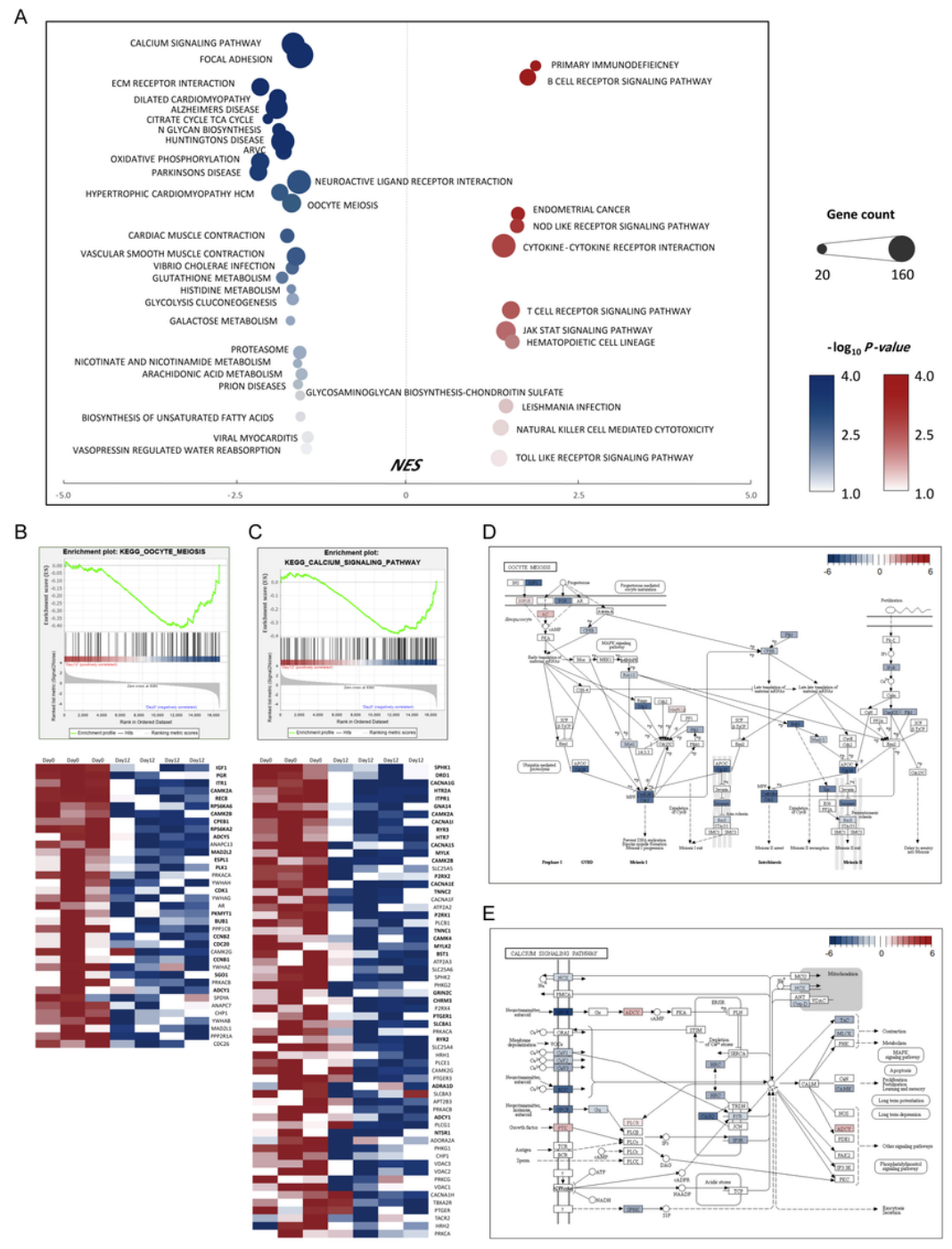

Figure 6

Gene Set Enrichment Analysis (GSEA) for comparisons of gene expression between Days 0 and 12. Scattered bubble plot from GSEA based on the KEGG database. The normalized enrichment score (NES) is represented on the $\mathrm{x}$-axis, and the bubble colour indicates the -log10 P-value. (A) The size of the bubble represents the number of genes counted in the terms. (B and C) A heatmap of the downregulated genes on Day 12 in the enriched pathways of oocyte meiosis and calcium signalling. (D and E) Changes in expression of DEG in the oocyte meiosis and calcium signalling pathways on Day 12.

\section{Supplementary Files}

This is a list of supplementary files associated with this preprint. Click to download.

- Additionalfile1.xlsx

- Additionalfile2.xIsx 